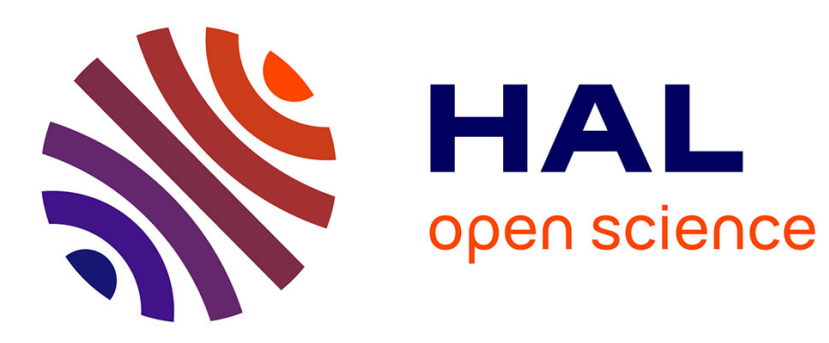

\title{
Linear combining in dependent $\alpha$-stable interference
}

Ce Zheng, Malcolm Egan, Laurent Clavier, Troels Pedersen, Jean-Marie Gorce

\section{To cite this version:}

Ce Zheng, Malcolm Egan, Laurent Clavier, Troels Pedersen, Jean-Marie Gorce. Linear combining in dependent $\alpha$-stable interference. ICC 2020 - IEEE International Conference on Communications, Jun 2020, Dublin, Ireland. pp.1-6, 10.1109/ICC40277.2020.9148724 • hal-02460193v2

\section{HAL Id: hal-02460193 \\ https://hal.science/hal-02460193v2}

Submitted on 16 Mar 2020

HAL is a multi-disciplinary open access archive for the deposit and dissemination of scientific research documents, whether they are published or not. The documents may come from teaching and research institutions in France or abroad, or from public or private research centers.
L'archive ouverte pluridisciplinaire $\mathbf{H A L}$, est destinée au dépôt et à la diffusion de documents scientifiques de niveau recherche, publiés ou non, émanant des établissements d'enseignement et de recherche français ou étrangers, des laboratoires publics ou privés. 


\title{
Linear Combining in Dependent $\alpha$-Stable Interference
}

\author{
Ce Zheng, Malcolm Egan, Laurent Clavier, Troels Pedersen and Jean-Marie Gorce
}

\begin{abstract}
Recently, there has been a proliferation of wireless communication technologies in unlicensed bands for the Internet of Things. A key question is whether these networks can coexist given that they have different power levels, symbol periods, and access protocols. The main challenge is to characterize the impact of mutual interference arising from distinct uncoordinated networks. It is known that when interferers form a homogeneous Poisson point process and transmit only on a single subband, the interference is often well-modeled by the heavy-tailed $\alpha$-stable distribution. In this paper, we focus on the scenario where interferers transmit on multiple subbands. Under a policy where each interferer independently accesses each band with probability $p$, we provide an exact characterization of the interference random vector. Exploiting this characterization, we derive optimal linear combining weights and an analytical approximation for the bit error rate (BER), accurate for large transmit power. A key observation is that the expression for the BER admits an interpretation in terms of an array gain and a fractional diversity gain.
\end{abstract}

\section{INTRODUCTION}

As the scale of wireless communication networks for the Internet of Things (IoT) is increasing, a fundamental challenge is interference management. Unlike devices in mobile cellular networks, there is very limited coordination within IoT networks. As a consequence, transmissions are only constrained by guidelines for the duty cycle or the time to listen in carriersense multiple access (CSMA) protocols [1]. As such, it is desirable for devices to perform their own interference mitigation through signal processing tailored to the interference statistics.

To this end, there has recently been significant work attempting to find appropriate statistical models for the interference. From the experimental side, a key observation is that the interference power on each subband is in fact heavy tailed [1], with the probability of large interference power significantly higher than for Gaussian models. This experimental observation is consistent with theoretical analysis of interference arising from a Poisson spatial field of interferers [2], [3] and variations including the Poisson-Poisson clustered interferers in [4]. In particular, $\alpha$-stable models have been shown to be a good approximation of the statistics for the interference amplitude when guard zones are sufficiently small and the network radius is sufficiently large [4]-[6].

C. Zheng and L. Clavier are with Université de Lille 1, IEMN UMR CNRS 8520, IRCICA USR CNRS 3380, Villeneuve d'Ascq, France. M. Egan and J.M. Gorce are with Univ. Lyon, INSA Lyon, INRIA, CITI, France. L. Clavier is also with IMT Lille Douai, France. T. Pedersen is with Department of Electronic Systems (WCN Section), Aalborg University, Denmark. This work has been (partly) funded by the French National Agency for Research (ANR) under grant ANR-16-CE25-0001 - ARBURST and the COST IRACON Inclusive Radio Communication Networks for $5 \mathrm{G}$ and Beyond.
Despite this extensive study of the interference on individual subbands, the random vector arising from interference on multiple subbands has received significantly less attention. While the marginal distribution for the interference on each subband remains $\alpha$-stable, the key difficulty is to characterize the statistical dependence between the interference on multiple subbands. Unlike Gaussian models where this dependence is completely characterized by the correlation, when the interference on each subband is $\alpha$-stable the correlation is infinite or undefined. As such, it is necessary to develop appropriate generalizations; e.g., via copulas [7], [8]. While copula models are very flexible and admit efficient estimation and simulation procedures, they are not often amenable to performance analysis. In particular, the impact of the dependence on system performance-i.e., achievable rates or uncoded bit error rate (BER) - is not well understood.

In this paper, we study the BER for a single transmitterreceiver pair exploiting linear combining in the presence of non-Gaussian interference. In particular, we adopt the same system model with multiple subbands as in [8] — based on interferer locations governed by a homogeneous Poisson point process. In this system model, each device accesses each subband independently with a given probability $p$. The extreme case $p \rightarrow 0$ corresponds to independent $\alpha$-stable interference on each subband, for which optimal linear combining has been studied in [9], [10]. The other extreme case $(p=1)$ studied in [8] corresponds to sub-Gaussian $\alpha$-stable interference random vectors. In this case, we show that the optimal combiner is maximum ratio combining (MRC), which is well-known to also arise in Gaussian noise. However, the BER behaves very differently. In fact, we show that for sufficiently large transmit power, the BER decays proportionally to $P^{-2 / \eta}$, where $P$ is the transmit power and $\eta$ is the path loss exponent.

We then turn to the general case $0<p<1$, for which we previously showed that the interference statistics are often well approximated by an interference random vector described by $\alpha$-stable marginals and dependence governed by a $t$-copula. In this paper, we provide an exact characterization of the interference random vector and show that it is, in fact, $\alpha$ stable as a random vector. Using this exact characterization, we derive the optimal combiner and characterize the BER in the high transmit power or "low interference" regime. We show that the BER admits an interpretation in terms of a fractional diversity order and an array gain dependent on the statistical dependence between interference on distinct subbands.

To verify our analysis, we perform Monte Carlo simulations which demonstrate the accuracy of the approximations for the BER. Moreover, the MRC outperforms equal gain combining 
(EGC) for all values of $p$. However, MRC is only optimal for $p=1$ and not in general for $0<p<1$. These results provide the first characterization of the impact of statistical dependence in $\alpha$-stable interference models. Our initial conclusions show that such dependence must be carefully accounted for in estimating the performance of IoT communication networks.

\section{A. Notation}

Vectors are denoted by bold lowercase letters and random vectors by bold uppercase letters, respectively (e.g., $\mathbf{x}, \mathbf{X}$ ). We denote the distribution of a random vector $\mathbf{X}$ by $P_{\mathbf{X}}$. If $\mathbf{X}, \mathbf{Y}$ are two random vectors equal in distribution, then we write $\mathbf{X} \stackrel{d}{=} \mathbf{Y}$. Let $f: \mathbb{R} \rightarrow \mathbb{R}$ and $g: \mathbb{R} \rightarrow \mathbb{R}$. We use the Landau notation where $f(x)=o(g(x))$ if $\lim _{x \rightarrow \infty} f(x) / g(x)=0$.

The family of $\alpha$-stable random vectors plays a key role in this paper and for completeness, we have provided relevant definitions and results in Appendix A.

\section{SySTEM MODEL}

\section{A. Signaling Scheme}

Consider a single antenna transmitter and single antenna receiver that communicate over multiple orthogonal frequency subbands. We suppose that the transmitter-receiver pair operates in the presence of a large number of other devices, which are neither coordinated with the transmitter nor the receiver.

The transmitter seeks to send a binary symbol $x \in$ $\{+1,-1\}$. Given a symbol $x$, the receiver observes an output $\mathbf{y} \in \mathbb{R}^{K}$ defined by

$$
\mathbf{y}=\mathbf{h} x+\mathbf{z}+\mathbf{n}
$$

where $\mathbf{h} \in \mathbb{R}^{K}$ corresponds to channel fading and $\mathbf{z} \in \mathbb{R}^{K}$ is interference in a set of $K$ orthogonal subbands. The channel fading is assumed to be perfectly known to the receiver; e.g., using pilots to estimate channel state information. The statistical model for the interference $\mathbf{z}$ is detailed in the sequel. The interference $\mathbf{z}$ is assumed to be a random vector admitting a probability density function (PDF). This implies that $\mathbf{y}$ also admits a PDF $f(\mathbf{y})$. We further assume that the thermal noise has a negligible impact on the distribution of $\mathbf{z}+\mathbf{n}$ and can be ignored.

This model is motivated by communication in the IoT, where low complexity devices typically exploit very simple communication schemes. The real-valued model arises in the case where signaling is only performed on either the in-phase or quadrature components. Another scenario where this model can arise is when the same symbol is sent over both the inphase and quadrature components and the received signal is viewed as the vector obtained by stacking the in-phase and quadrature components for each subband.

Given the observation $\mathbf{y}$ and equally likely symbols (i.e., +1 and -1 ), the BER is minimized by the likelihood ratio test

$$
\Lambda(\mathbf{y})=\frac{f(\mathbf{y} \mid x=1)}{f(\mathbf{y} \mid x=-1)} \stackrel{x=1}{\gtrless} 1 .
$$

As will be explained further in Section II-B, the likelihoods in (2) do not admit tractable closed-form solutions. As such, a statistic obtained from linear combining is considered instead, and the resulting linear detection rule is then given by

$$
\tilde{y}=\mathbf{w}^{T} \mathbf{y} \underset{x=-1}{\gtrless} 0 .
$$

where the weights $\mathbf{w} \in \mathbb{R}^{K}$ satisfy $\|\mathbf{w}\|=1$.

\section{B. Interference Model}

Consider a network of interfering devices located according to a homogeneous Poisson point process (PPP) $\Phi$ with intensity $\lambda$. These devices form interferers for a receiver located at the origin. We assume that the same frequency band is shared by all devices, and that this band is also the one utilized by the desired transmitter-receiver pair. For example, this is the case for the frequency band $863 \mathrm{MHz}$ to $870 \mathrm{MHz}$ used for low power wide area networks (LPWAN).

The frequency band is divided into $K$ subbands. In a given time slot for the desired transmitter-receiver pair, each interfering device transmits on a subset of the subbands. In particular, each interfering device scans all subbands, selecting each subband independently with probability $p$ (interpreted as a function of the quantity of data that interfering devices seek to transmit to their respective access points). As a consequence, the probability that a given interfering device transmits on $k$ subbands is given by $\left(\begin{array}{c}K \\ k\end{array}\right) p^{k}(1-p)^{K-k}$. As the interfering devices are also assumed to be uncoordinated, the subbands selected by a given device $l \in \Phi$ are independent of the subbands selected by any other device $l^{\prime} \in \Phi, l^{\prime} \neq l$. The set of devices in $\Phi$ that transmit on the $i$-th subband are denoted by $\Phi_{i}$.

The interference observed by the desired receiver (corresponding to the transmitter-receiver pair in Section II-A) on $i$-th subband is given by

$$
z_{i}=\sum_{j \in \Phi_{i}} r_{j}^{-\eta / 2} x_{j, i}, \quad i \in\{1, \cdots, K\}
$$

where $r_{j}$ is the distance from device $j$ in $\Phi_{i}$ to the desired receiver, $\eta$ is the path loss exponent, and $x_{j, i} \in \mathbb{R} \sim \mathcal{N}\left(0, \sigma_{I}^{2}\right)$ corresponds to the combination of baseband emission and small-scale fading. Under Rayleigh fading, a Gaussian approximation for $x_{j, i}$ can well approximate the true statistics [3].

After stacking the interference on each subband, the resulting interference random vector is given by

$$
\mathbf{z}=\left(z_{1}, \ldots, z_{K}\right)^{T},
$$

corresponding to the interference term in (1). By the independent thinning theorem for homogeneous Poisson point processes, it follows that each $\Phi_{i}$ also form homogeneous Poisson point processes. As a consequence, the interference on each subband in our model is non-Gaussian; namely, $\alpha$ stable distributed for which relevant definitions and results are provided in Appendix A. This is detailed in the following theorem given, for example, in [7].

Theorem 1. Consider the interference on subband $i$, denoted by $z_{i}$ in (4). Suppose that the baseband emissions $x_{j, i} \sim$ $\mathcal{N}\left(0, \sigma_{I}^{2}\right)$ are also i.i.d. Then, $z_{i}$ in (4) converges almost surely to a symmetric $4 / \eta$-stable random variable with the scale parameters given by 


$$
\gamma_{z_{i}}=\left(\pi \lambda p C_{\frac{4}{\eta}}^{-1} \mathbb{E}\left[\left|x_{j, i}\right|^{\frac{4}{\eta}}\right]\right)^{\frac{\eta}{4}},
$$

where

$$
C_{\alpha}=\left\{\begin{array}{ll}
\frac{1-\alpha}{\Gamma(2-\alpha) \cos (\pi \alpha / 2)}, & \text { if } \alpha \neq 1 \\
2 / \pi, & \text { if } \alpha=1 .
\end{array} .\right.
$$

\section{Optimal Linear Receiver for Sub-Gaussian $\alpha$-STABLE INTERFERENCE}

Our focus in the remainder of the paper is to obtain optimal linear combiners and to characterize the BER. While this problem has been widely studied in the case of Gaussian noise and for i.i.d. symmetric $\alpha$-stable noise [9], [10], little is presently known about the case of non-trivial probabilistic dependence. Preliminary definitions and characterizations of $\alpha$-stable random vectors are presented in Appendix A.

An important special case of the model detailed in Section II is when each interferer selects each subband with probability one; that is, $\Phi_{i}=\Phi, i=1, \ldots, K$ almost surely. It is known from Theorem 3 in [7] that the resulting interference random vector $\mathbf{z}$ is sub-Gaussian $\alpha$-stable, defined as follows.

Definition 1. Any vector $\mathbf{X}$ distributed as $\mathbf{X}=$ $\left[A^{1 / 2} G_{1}, \ldots, A^{1 / 2} G_{d}\right]^{T}$ is called a sub-Gaussian $\alpha$-stable random vector in $\mathbb{R}^{d}$ with underlying Gaussian vector $\mathbf{G}$ if it satisfies

$$
A \sim S_{\alpha / 2}\left(\left(\cos \frac{\pi}{4} \alpha\right)^{2 / \alpha}, 1,0\right),
$$

where $A$ and $\mathbf{G}$ are independent. If $\mathbf{G}=\left[G_{1}, \ldots, G_{d}\right]^{T} \sim$ $\mathcal{N}\left(0, \sigma^{2} \mathbf{I}\right)$, then $X_{i} \sim S_{\alpha}(\gamma, 0,0)$ where $\gamma=\sigma / \sqrt{2}$ and $\sigma$ is called the parameter of $\mathbf{X}$.

\section{A. Optimal Linear Combiner}

We now derive the optimal linear combiner when the interference random vector $\mathbf{z}$ is sub-Gaussian $\alpha$-stable. The BER can be expressed by

$$
P_{e}(\mathbf{w})=\frac{1}{2}\left[\mathbb{P}\left(\mathbf{w}^{T} \mathbf{y}>0 \mid x=-1\right)+\mathbb{P}\left(\mathbf{w}^{T} \mathbf{y} \leq 0 \mid x=1\right)\right] .
$$

Using the detection rule in (3), it follows that

$$
\begin{aligned}
\mathbb{P}\left(\mathbf{w}^{T} \mathbf{y}>0 \mid x=-1\right) & =\mathbb{P}\left(-\mathbf{w}^{T} \mathbf{h}+\mathbf{w}^{T} \mathbf{z}>0\right) \\
& =\mathbb{P}\left(\mathbf{w}^{T} \mathbf{z}>\mathbf{w}^{T} \mathbf{h}\right),
\end{aligned}
$$

and

$$
\begin{aligned}
\mathbb{P}\left(\mathbf{w}^{T} \mathbf{y} \leq 0 \mid x=1\right) & =\mathbb{P}\left(\mathbf{w}^{T} \mathbf{h}+\mathbf{w}^{T} \mathbf{z} \leq 0\right) \\
& =\mathbb{P}\left(\mathbf{w}^{T} \mathbf{z} \geq \mathbf{w}^{T} \mathbf{h}\right),
\end{aligned}
$$

since $\mathbf{z}$ is a sub-Gaussian $\alpha$-stable, a special case of a symmetric $\alpha$-stable random vector, $\mathbf{w}^{T} \mathbf{z}$ is symmetric $\alpha$-stable.

Theorem 2. Let $\mathbf{z}$ be a symmetric $\alpha$-stable random vector with underlying Gaussian vector $\mathbf{G} \sim \mathcal{N}\left(0, \sigma_{\mathbf{z}} \mathbf{I}\right)$ and parameter $\sigma_{\mathbf{z}}>0$. Then, the optimal weight vector $\mathrm{w}$ minimizing the BER in (9) is given by

$$
\mathbf{w}=\mathbf{h} /\|\mathbf{h}\| .
$$

where $\|\mathbf{h}\|$ is the Euclidean norm of $\mathbf{h}$. That is, the optimal linear combiner is maximal ratio combining.

Proof. We first establish that where

$$
P_{e}(\mathbf{w})=\mathbb{P}\left(\tilde{z}>\mathbf{w}^{T} \mathbf{h} /\|\mathbf{w}\|\right),
$$

$$
\tilde{z}=\mathbf{w}^{\mathbf{T}} \mathbf{z} /\|\mathbf{w}\| \sim \mathcal{S}_{\alpha}\left(\gamma_{\mathbf{z}}, 0,0\right), \quad \gamma_{\mathbf{z}}=\sigma_{\mathbf{z}} / \sqrt{2} .
$$

Since $\mathbf{z}$ is sub-Gaussian $\alpha$-stable with parameter $\sigma_{\mathbf{z}}$, it admits the scale-mixture representation

$$
\mathbf{z}=A^{\frac{1}{2}}\left(G_{1}, G_{2}, \ldots, G_{K}\right)^{T},
$$

where $A \sim S_{\alpha / 2}\left(\left(\cos \frac{\pi}{4} \alpha\right)^{2 / \alpha}, 1,0\right)$ and $G_{k} \sim$ $\mathcal{N}\left(0, \sigma_{\mathbf{z}}\right), k=1, \ldots, K$, all independent. As such,

$$
\mathbf{w}^{T} \mathbf{z}=A^{\frac{1}{2}} \sum_{k=1}^{K} w_{k} G_{k} \stackrel{d}{=} A^{\frac{1}{2}} \tilde{G},
$$

where $\tilde{G} \sim \mathcal{N}\left(0, \sigma_{\mathbf{z}}^{2} \sum_{k=1}^{K} w_{k}^{2}\right)$, which after dividing by $\|\mathbf{w}\|$ yields (14).

By the fact that $\tilde{z} \sim \mathcal{S}_{\alpha}\left(\gamma_{\mathbf{z}}, 0,0\right)$-i.e., the parameters are independent of $\mathbf{w}$-in (13) and the cumulative distribution of $\tilde{z}$ is non-decreasing, it follows that minimizing $P_{e}(\mathbf{w})$ is equivalent to maximizing $\mathbf{w}^{T} \mathbf{h} /\|\mathbf{w}\|$.

Applying the Cauchy-Schwarz inequality then yields

$$
\left|\mathbf{w}^{T} \mathbf{h}\right|^{2} \leq\|\mathbf{w}\|^{2}\|\mathbf{h}\|^{2}
$$

Then, equality holds if and only if $\mathbf{w}$ and $\mathbf{h}$ are linearly dependent; i.e., $\mathbf{w}=c \mathbf{h}$, for some $c>0$. Setting $c=1 /\|\mathbf{h}\|$ to satisfy the constraint $\|\mathbf{w}\|=1$, the equality (12) is obtained.

\section{B. Bit Error Rate}

We now characterize the BER in the case the interference random vector is sub-Gaussian $\alpha$-stable. The basis of the analysis is the following property of symmetric $\alpha$-stable random variables, which can be found in [11].

Lemma 1. Let $X \sim S_{\alpha}(\gamma, 0,0)$. Then as $b \rightarrow \infty$,

$$
\mathbb{P}(X>b)=\frac{1}{2} C_{\alpha} \gamma^{\alpha} b^{-\alpha}+o\left(b^{-\alpha}\right),
$$

where $C_{\alpha}$ is given in (7).

It is now straightforward to characterize the BER when $\mathbf{z}$ is sub-Gaussian $\alpha$-stable.

Theorem 3. Let $\mathbf{z}$ be a K-dimensional sub-Gaussian $\alpha$-stable random vector with parameter $\sigma_{\mathbf{z}}$ and the linear combining weights be $\mathbf{w} \in \mathbb{R}^{K}$. Then, as $\|\mathbf{h}\| \rightarrow \infty$,

$$
P_{e}(\mathbf{w})=\frac{1}{2} C_{\alpha} \gamma_{\mathbf{z}}^{\alpha}\left(\mathbf{w}^{T} \mathbf{h} /\|\mathbf{w}\|\right)^{-\alpha}+o\left(\left(\mathbf{w}^{T} \mathbf{h} /\|\mathbf{w}\|\right)^{-\alpha}\right) .
$$

where $\gamma_{\mathbf{z}}=\sigma_{\mathbf{z}} / \sqrt{2}$. Moreover, the optimal linear weights admit a BER

$$
P_{e}(\mathbf{h})=\frac{1}{2} C_{\alpha} \gamma_{\mathbf{z}}^{\alpha}\|\mathbf{h}\|^{-\alpha}+o\left(\|\mathbf{h}\|^{-\alpha}\right) .
$$

Proof. Use Lemma 1 in (13).

One observation is that the form of the BER in (19) bears striking similarities to the BER of linear combining in Rayleigh fading with Gaussian noise. In particular, the exponent $\alpha$ plays a role similar to a fractional diversity gain with the key difference from the Rayleigh fading scenario that it lies in $0<\alpha<2$. Similarly, the factor $\frac{1}{2} C_{\alpha} \gamma_{\mathbf{z}}^{\alpha}$ plays the role of an array gain. 


\section{General Interference Model}

We now turn to the general interference model detailed in Section II, focusing on the case $K=2$ due to space constraints (similar analysis applies for $K>2$.) We first establish that the interference random vector remains symmetric $\alpha$-stable. This is non-trivial as, unlike the Gaussian noise case, a random vector with symmetric $\alpha$-stable marginals is not necessarily symmetric $\alpha$-stable as a random vector, which is formally defined in Appendix A. We then develop new bounds on the error probability.

\section{A. Interference Statistics}

A general exact characterization of the interference random vector $\mathbf{z}$ in (5) is given in the following theorem.

Theorem 4. The interference random vector $\mathbf{z} \in \mathbb{R}^{2}$ in (5) has characteristic function

$$
\begin{aligned}
\Phi_{\mathbf{z}}(\boldsymbol{\theta}) & =\mathbb{E}\left[e^{i\left(\theta_{1} z_{1}+\theta_{2} z_{2}\right)}\right] \\
& =\exp \left(i \gamma_{1}^{\alpha}\left|\theta_{1}^{2}+\theta_{2}^{2}\right|^{\frac{\alpha}{2}}+i \gamma_{2}^{\alpha}\left|\theta_{1}\right|^{\alpha}+i \gamma_{2}^{\alpha}\left|\theta_{2}\right|^{\alpha}\right)
\end{aligned}
$$

where

$$
\begin{aligned}
& \gamma_{1}=\sigma_{I}\left(\pi \lambda p^{2} C_{4 / \eta}^{-1} \mathbb{E}\left[\left|Z_{0}\right|^{4 / \eta}\right]\right)^{\eta / 4} \\
& \gamma_{2}=\sigma_{I}\left(\pi \lambda p(1-p) C_{4 / \eta}^{-1} \mathbb{E}\left[\left|Z_{0}\right|^{4 / \eta}\right]\right)^{\eta / 4}
\end{aligned}
$$

with $Z_{0} \sim \mathcal{N}(0,1)$. That is, $\mathbf{z}$ is a symmetric $\alpha$-stable random vector with spectral measure on $\mathbb{S}^{1}$ given by $\Gamma=\Gamma_{1}+\Gamma_{2}$, with $\Gamma_{1}$ uniform on $\mathbb{S}^{1}$ and $\Gamma_{2}$ concentrated on $( \pm 1,0),(0, \pm 1)$.

Proof. Let $\Phi$ be a homogeneous Poisson point process with intensity $\lambda$. Under the model in Section II-B, the interference on each subband is given by

$$
\begin{aligned}
& z_{1}=\sum_{j \in \Phi_{1}} r_{j}^{-\eta / 2} x_{j, 1}, \\
& z_{2}=\sum_{j \in \Phi_{2}} r_{j}^{-\eta / 2} x_{j, 2} .
\end{aligned}
$$

where $x_{j, i}, i=1,2$ is defined in (4). Since each device independently chooses to access each subband $B_{i}, i=1,2$ with probability $p$, it follows that the processes $\Phi_{1} \cup \Phi_{2}, \Phi_{1} \backslash \Phi_{2}$ and $\Phi_{2} \backslash \Phi_{1}$ are independent Poisson point processes with intensities, respectively,

$$
\begin{aligned}
& \lambda_{1}=p^{2} \lambda \\
& \lambda_{2}=\lambda_{3}=p(1-p) \lambda
\end{aligned}
$$

The characteristic function $\Phi_{\mathbf{z}}(\boldsymbol{\theta})$ is then given by

$$
\begin{aligned}
\Phi_{\mathbf{z}}(\boldsymbol{\theta}) & =\mathbb{E}\left[e^{i\left(\theta_{1} z_{1}+\theta_{2} z_{2}\right)}\right] \\
& =\exp \left(i \left(\sum_{k_{1} \in \Phi_{1} \cup \Phi_{2}} r_{k_{1}}^{-\eta / 2}\left(\theta_{1} x_{k_{1}, 1}+\theta_{2} x_{k_{1}, 2}\right)\right.\right. \\
& \left.\left.+\sum_{k_{2} \in \Phi_{1} \backslash \Phi_{2}} r_{k_{2}}^{-\eta / 2} \theta_{1} x_{k_{2}, 1}+\sum_{k_{3} \in \Phi_{2} \backslash \Phi_{1}} r_{k_{3}}^{-\eta / 2} \theta_{2} x_{k_{3}, 2}\right)\right)
\end{aligned}
$$

Noting that $\left(r_{k_{j}}^{2}\right)$ are one-dimensional Poisson point processes with parameters $\pi \lambda_{j}$ [2] and using the LePage series representation of symmetric $\alpha$-stable random variables [11, Corollary 1.4.3], it follows that

$$
\begin{aligned}
V_{1} & =\sum_{k_{1} \in \Phi_{1} \cup \Phi_{2}} r_{k_{1}}^{-\eta / 2}\left(\theta_{1} x_{k_{1}, 1}+\theta_{2} x_{k_{1}, 2}\right) \\
V_{2} & =\sum_{k_{2} \in \Phi_{1} \backslash \Phi_{2}} r_{k_{2}}^{-\eta / 2} \theta_{1} x_{k_{2}, 1} \\
V_{3} & =\sum_{k_{3} \in \Phi_{2} \backslash \Phi_{1}} r_{k_{3}}^{-\eta / 2} \theta_{2} x_{k_{3}, 2}
\end{aligned}
$$

are each independent symmetric $\alpha$-stable random variables. As such, $V_{1}+V_{2}+V_{3}=\theta_{1} z_{1}+\theta_{2} z_{2}$ is also a symmetric $\alpha$-stable random variable, irrespective of the choice $\boldsymbol{\theta} \in \mathbb{R}^{2}$. In particular, let $Z_{0} \sim \mathcal{N}(0,1)$, then

$$
\begin{aligned}
V_{1} & \sim \mathcal{S}_{4 / \eta}\left(\sigma_{I} \sqrt{\theta_{1}^{2}+\theta_{2}^{2}}\left(\pi \lambda p^{2} C_{4 / \eta}^{-1} \mathbb{E}\left[\left|Z_{0}\right|^{4 / \eta}\right]\right)^{\eta / 4}, 0,0\right) \\
V_{2} & \sim \mathcal{S}_{4 / \eta}\left(\sigma_{I}\left|\theta_{1}\right|\left(\pi \lambda p(1-p) C_{4 / \eta}^{-1} \mathbb{E}\left[\left|Z_{0}\right|^{4 / \eta}\right]\right)^{\eta / 4}, 0,0\right) \\
V_{3} & \sim \mathcal{S}_{4 / \eta}\left(\sigma_{I}\left|\theta_{2}\right|\left(\pi \lambda p(1-p) C_{4 / \eta}^{-1} \mathbb{E}\left[\left|Z_{0}\right|^{4 / \eta}\right]\right)^{\eta / 4}, 0,0\right) .
\end{aligned}
$$

By Theorem 2.1.5 in [11], it then follows that $\mathbf{z}$ is a symmetric $\alpha$-stable random vector with characteristic function given by (21).

As expected, when $p \rightarrow 1$, we recover the sub-Gaussian $\alpha$-stable characteristic function studied in Section III.

\section{B. Optimal Linear Combining}

We now study the BER for the general interference statistics in Theorem 4.

Theorem 5. Let $\mathbf{z}$ have the characteristic function given in Theorem 4, corresponding to the general interference model in Section II-B. Then, the optimal combining weights are the solution of

$$
\max _{\mathbf{w} \in \mathbb{R}^{2}:\|\mathbf{w}\|=1} \frac{\mathbf{w}^{T} \mathbf{h}}{\left(\gamma_{1}^{\alpha}+\gamma_{2}^{\alpha}\left|w_{1}\right|^{\alpha}+\gamma_{2}^{\alpha}\left|w_{2}\right|^{\alpha}\right)^{1 / \alpha}} .
$$

Proof. The BER is given by

$$
P_{e}=\mathbb{P}\left(w_{1} z_{1}+w_{2} z_{2}>w_{1} h_{1}+w_{2} h_{2}\right) .
$$

Now,

$$
\begin{aligned}
& w_{1} z_{1}+w_{2} z_{2}=\sum_{k \in \Phi_{1} \cap \Phi_{2}} r_{k}^{-\eta / 2}\left(w_{1} X_{k, 1}+w_{2} X_{k, 2}\right) \\
& +\sum_{k \in \Phi_{1} \backslash \Phi_{2}} w_{1} r_{k}^{-\eta / 2} X_{k, 1}+\sum_{k \in \Phi_{2} \backslash \Phi_{1}} w_{2} r_{k}^{-\eta / 2} X_{k, 1} .
\end{aligned}
$$

Suppose that $X_{k, j} \sim \mathcal{N}\left(0, \sigma_{I}^{2}\right)$ and call the three terms above $V_{1}, V_{2}, V_{3}$, respectively. Then,

$$
\begin{aligned}
& V_{1} \sim S_{\alpha}\left(\gamma_{1} \sqrt{w_{1}^{2}+w_{2}^{2}}, 0,0\right) \\
& V_{2} \sim S_{\alpha}\left(\gamma_{2}\left|w_{1}\right|, 0,0\right) \\
& V_{3} \sim S_{\alpha}\left(\gamma_{2}\left|w_{2}\right|, 0,0\right)
\end{aligned}
$$


As such,

$$
\begin{aligned}
& w_{1} z_{1}+w_{2} z_{2} \\
& \sim S_{\alpha}\left(\left(\gamma_{1}^{\alpha}\left(w_{1}^{2}+w_{2}^{2}\right)^{\alpha / 2}+\gamma_{2}^{\alpha}\left|w_{1}\right|^{\alpha}+\gamma_{2}^{\alpha}\left|w_{2}\right|^{\alpha}\right)^{1 / \alpha}, 0,0\right) .
\end{aligned}
$$

Hence,

$$
P_{e}=\mathbb{P}\left(N>\frac{\mathbf{w}^{T} \mathbf{h}}{\left(\gamma_{1}^{\alpha}\left(w_{1}^{2}+w_{2}^{2}\right)^{\alpha / 2}+\gamma_{2}^{\alpha}\left|w_{1}\right|^{\alpha}+\gamma_{2}^{\alpha}\left|w_{2}\right|^{\alpha}\right)^{1 / \alpha}}\right),
$$

where $N \sim S_{\alpha}(1,0,0)$. An examination of (34), reveals that scaling $\mathbf{w}$ does not affect the BER. Without loss of generality, we therefore set $\|\mathbf{w}\|=1$. Under this condition $w_{1}^{2}+w_{2}^{2}=1$ and the optimal weights are given by (29).

Using the definitions of $\gamma_{1}, \gamma_{2}$ in Theorem 4, observe that for $p \rightarrow 1$ we recover the result in Theorem 2. A similar argument as in Theorem 3 then also gives a characterization of the BER for large $\|\mathbf{h}\|$.

Theorem 6. Let $\mathbf{z}$ be a symmetric $\alpha$-stable random vector with characteristic function in Theorem 4, and linear combining weights be $\mathbf{w} \in \mathbb{R}^{K}$. Then, as $\|\mathbf{h}\| \rightarrow \infty$,

$$
\begin{gathered}
P_{e}(\mathbf{w})=\frac{1}{2} C_{\alpha} \gamma_{\mathbf{w}}^{\alpha}\left(\mathbf{w}^{T} \mathbf{h} /\|\mathbf{w}\|\right)^{-\alpha}+o\left(\left(\mathbf{w}^{T} \mathbf{h} /\|\mathbf{w}\|\right)^{-\alpha}\right) . \\
\text { where } \gamma_{\mathbf{w}}=\left(\gamma_{1}^{\alpha}\left(w_{1}^{2}+w_{2}^{2}\right)^{\alpha / 2}+\gamma_{2}^{\alpha}\left|w_{1}\right|^{\alpha}+\gamma_{2}^{\alpha}\left|w_{2}\right|^{\alpha}\right)^{1 / \alpha} . \\
\text { V. NUMERICAL RESULTS }
\end{gathered}
$$

\section{Numerical Results}

In this section, we validate our analysis by studying the Monte Carlo simulations of the BER. To study the BER in the presence of sub-Gaussian $\alpha$-stable interference, as considered in Section III, we set $\gamma_{\mathbf{z}}=1, \alpha=4 / 5$ and $x= \pm 1$. The channel vector is

$$
\begin{gathered}
\mathbf{h}=\sqrt{\rho} \times[0.0949,0.3237,0.3988,0.1522,0.0563,0.2308 \\
0.0765,0.0605,0.6317,0.4889],
\end{gathered}
$$

where $\|\mathbf{h}\|^{2}=\rho$. The system behavior under this choice of channel vector is representative, validated through an extensive simulation study for other choices of $\mathbf{h}$. While it is also possible to study the average behavior under, for example, Rayleigh fading, the choice of a fixed $\mathbf{h}$ enables a direct comparison with the analysis in the previous sections.

Fig. 1 plots BER for varying $\|\mathbf{h}\|^{2}=\rho$ and both the optimal combiner, i.e., MRC (by Theorem 2) and EGC. As expected from Theorem 2, the MRC combiner outperforms EGC. Moreover, the asymptotic approximation for the BER in Theorem 3 is in good agreement with the Monte Carlo simulation for sufficiently large $\|\mathbf{h}\|$.

To validate the model is consistent with Theorem 4, we plot the samples simulated from the system model and samples generated from (21) in Fig. 2 and the corresponding joint PDFs in Fig. 3. The channel is fixed again to be (36). Observe that the samples and the joint PDF from the model in Theorem 4 are consistent with the samples and the joint PDF from the system model detailed in Section II.

Fig. 4 plots the BER for varying $p$. Optimal linear combining (OLC) based on Theorem 5, MRC and EGC are considered. The BER for $p \approx 1$ under OLC and MRC approximately equal, which further validates Theorem 2 that

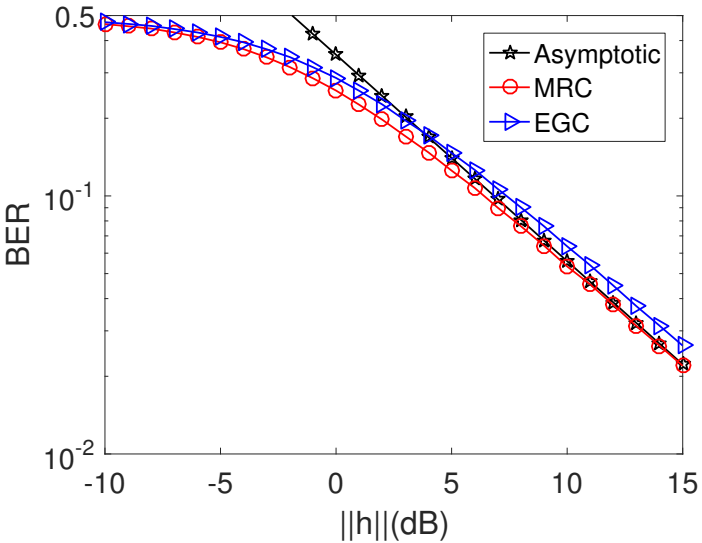

Fig. 1: Bit error rates comparison of MRC and EGC for subGaussian $\alpha$-stable interference under different $\|h\|$ with $K=$ 10 channels, $\gamma_{\mathbf{z}}=1, \alpha=4 / 5$ and $x= \pm 1$

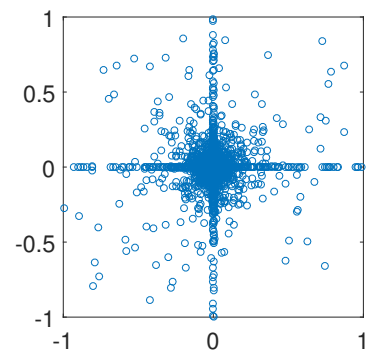

(a) System Model.

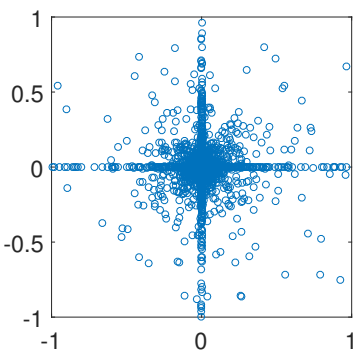

(b) Model in (21).
Fig. 2: Scatter Plots of the interference random vector with $p=0.5, \lambda=0.001 \mathrm{~m}^{-2}, \eta=5$, and $\sigma_{I}=1$.

the optimal combiner for sub-Gaussian $\alpha$-stable interference is MRC.

In Fig. 4, low values of $p$ imply that each device transmits with a low probability, consistent with the low BER observed in the figure. Increasing $p$ changes the statistics of interference vector in two ways. First, it increases the scale parameter of each marginals leading to a higher BER. Second, the interference vector becomes increasingly dependent and approximately sub-Gaussian when $p \approx 1$, which-for equal scale parameters-reduces the BER. In particular, for the sub-

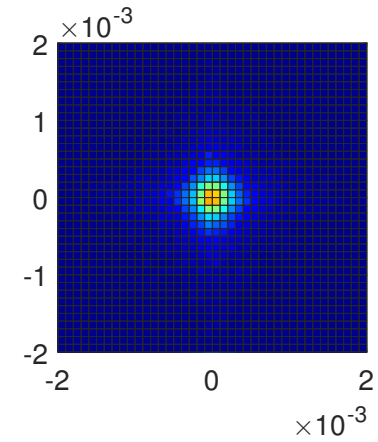

(a) System Model.

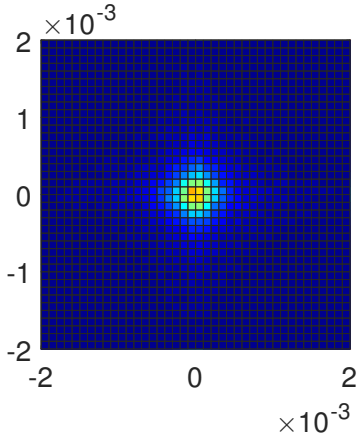

(b) Model in (21).
Fig. 3: Probability density functions of the interference random vector with settings as in Fig. 2. 


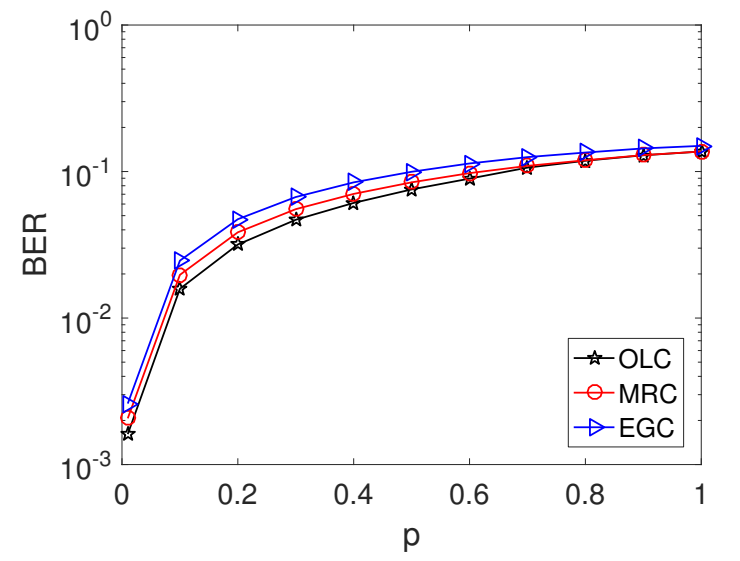

Fig. 4: Bit error rates of OC, MRC and EGC under different $p$ with $\lambda=0.001 \mathrm{~m}^{-2},\|\mathbf{h}\|=10^{-3}, \eta=5$, and $\sigma_{I}=1$.

Gaussian $\alpha$-stable and independent $\alpha$-stable vector with same marginal distributions, the scale parameter $\gamma_{\mathbf{w}}$ of the weighted sum of the interference on each subband is different. Observe that $\gamma_{\mathbf{w}}$ for sub-Gaussian is less than $\gamma_{\mathbf{w}}$ for independent case. That is, $w_{1} z_{1}+w_{2} z_{2}, w_{1}=w_{2}=1$ and $z_{i} \sim S_{\alpha}(1,0,0)$, $\gamma_{\mathbf{w}}=\sqrt{2}$ for sub-Gaussian and $\gamma_{\mathbf{w}}=2^{1 / \alpha}$ for independent subbands. We conclude from Fig. 4 that the impact on the BER of the scale parameter dominates the impact of dependence as $p$ increases.

\section{CONCLUSION}

Both experiments and theory have recently suggested that interference in the IoT is non-Gaussian and often heavy tailed for a single subband. In this paper, we have studied an interference model with multiple subbands which accounts for probabilistic dependence between the interference on each subband. For a general access model, we established an exact characterization of the interference random vector and an asymptotic approximation of the BER. This provides a basis for further investigation into the impact of statistical dependence of the interference random vector, including optimal receiver design and extending recent information theoretic results for $\alpha$-stable models [12].

\section{APPENDIX A}

THE $\alpha$-STABLE MODEL

The heavy-tailed probability density functions of the $\alpha$ stable model have been widely used to model impulsive signals [11], [13]. The PDF of an $\alpha$-stable random variable is described by four parameters: the characteristic exponent $0<\alpha \leq 2$; the scale parameter $\gamma \in \mathbb{R}_{+}$; the skew parameter $\beta \in[-1,1]$; and the shift parameter $\delta \in \mathbb{R}$. As such, a common notation for an $\alpha$-stable random variable $X$ is $X \sim S_{\alpha}(\gamma, \beta, \delta)$. In the case $\beta=\delta=0, X$ is said to be a symmetric $\alpha$-stable $(\mathrm{S} \alpha \mathrm{S})$ random variable.

In general, $\alpha$-stable random variables do not have closedform PDFs, but are usually represented by their characteristic function, given by [11, Eq. 1.1.6]

$\mathbb{E}\left[e^{i \theta X}\right]=\left\{\begin{array}{l}\exp \left\{-\gamma^{\alpha}|\theta|^{\alpha}\left(1-i \beta(\operatorname{sign} \theta) \tan \frac{\pi \alpha}{2}\right)+i \delta \theta\right\}, \alpha \neq 1 \\ \exp \left\{-\gamma|\theta|\left(1+i \beta \frac{2}{\pi}(\operatorname{sign} \theta) \log |\theta|\right)+i \delta \theta\right\}, \alpha=1\end{array}\right.$

Multivariate symmetric $\alpha$-stable variables can be defined as

Definition 2. A random vector $\mathbf{X}$ in $\mathbb{R}^{d}$ is symmetric $\alpha$ stable if for every $A, B>0$ there exists a $C>0$ such that

$$
A \mathbf{X}^{(1)}+B \mathbf{X}^{(2)} \stackrel{d}{=} C \mathbf{X}
$$

where $\mathbf{X}^{(1)}, \mathbf{X}^{(2)}$ are independent copies of $\mathbf{X}$.

Symmetric $\alpha$-stable random vectors can be represented via their characteristic function, given by [11, Theorem 2.4.3]

$$
\mathbb{E}\left[e^{i \boldsymbol{\theta} \cdot \mathbf{x}}\right]=\exp \left(-\int_{\mathbb{S}^{d-1}}\left|\sum_{k=1}^{d} \theta_{k} s_{k}\right|^{\alpha} \Gamma(d \mathbf{s})\right),
$$

where $\Gamma$ is the unique symmetric spectral measure on the surface of the $d$-dimensional unit sphere $\mathbb{S}^{d-1}$. Unlike Gaussian models, each element in $\mathbf{X}$ is an $\alpha$-stable random variable if $\mathbf{X}$ is an $\alpha$-stable vector, but not all random vectors with symmetric $\alpha$-stable marginals form symmetric $\alpha$-stable random vectors [11].

\section{REFERENCES}

[1] M. Lauridsen, B. Vejlgaard, I. Kovács, H. Nguyen, and P. Mogensen, "Interference measurements in the European $868 \mathrm{MHz}$ ISM band with focus on LoRa and SigFox," in IEEE Wireless Communications and Networking Conference (WCNC), 2017.

[2] J. Ilow and D. Hatzinakos, "Analytic alpha-stable noise modeling in a poisson field of interfers or scatterers," IEEE Transactions on Signal Processing, vol. 46, no. 6, pp. 1601-1611, 1998.

[3] P. Pinto and M. Win, "Communication in a Poisson field of interfererspart I: interference distribution and error probability," IEEE Transactions on Wireless Communications, vol. 9, no. 7, pp. 2176-2186, 2010.

[4] K. Gulati, B. Evans, J. Andrews, and K. Tinsley, "Statistics of co-channel interference in a field of Poisson-Poisson clustered interferers," IEEE Transactions on Signal Processing, vol. 58, no. 12, pp. 6207-6222, 2010.

[5] E. Sousa, "Performance of a spread spectrum packet radio network link in a Poisson field of interferers," IEEE Transactions on Information Theory, vol. 38, no. 6, pp. 1743-1754, 1992.

[6] C. Zheng, M. Egan, L. Clavier, G. W. Peters, and J.-M. Gorce, "On the Validity of Isotropic Complex $\alpha$-Stable Interference Models for Interference in the IoT," in GRETSI 2019 - Colloque francophone de traitement du signal et des images, (Lille, France), p. 4, Aug. 2019.

[7] M. Egan, L. Clavier, C. Zheng, M. de Freitas, and J.-M. Gorce, "Dynamic interference for uplink SCMA in large-scale wireless networks without coordination," EURASIP Journal on Wireless Communications and Networking, vol. 1, 2018.

[8] C. Zheng, M. Egan, L. Clavier, G. Peters, and J.-M. Gorce, "Copulabased interference models for iot wireless networks," in ICC 2019-53rd IEEE International Conference on Communications, pp. 1-6, 2019.

[9] S. Niranjayan and N. Beaulieu, "The BER optimal linear rake receiver for signal detection in symmetric alpha-stable noise," IEEE Transactions on Communications, vol. 57, pp. 3585-3588, Dec. 2009.

[10] S. Niranjayan and N. Beaulieu, "BER optimal linear combiner for signal detection in symmetric alpha-stable noise: small values of alpha," IEEE Transactions on Wireless Communications, vol. 9, pp. 886-890, Mar. 2010.

[11] G. Samorodnitsky and M. Taqqu, Stable Non-Gaussian Random Processes. New York, NY: CRC Press, 1994.

[12] M. de Freitas, M. Egan, L. Clavier, G. Peters, and N. Azzaoui, "Capacity bounds for additive symmetric alpha-stable noise channels," IEEE Transactions on Information Theory, vol. 63, pp. 5115-5123, Aug. 2017.

[13] C. Nikias and M. Shao, Signal processing with alpha-stable distributions and applications. New York, NY: Wiley, 1995. 